\title{
DELIRIUM POSTOPERATORIO PRECOZ AUMENTA EL RIESGO DE DESARROLLAR DELIRIUM TARDÍO EN PACIENTES MAYORES DE 65 AÑOS
}

\author{
Rodrigo Gutiérrez ${ }^{1}$, Fernando Reyes ${ }^{1}$, Ignacio Aranibar ${ }^{2}$, Makarena Contreras ${ }^{4}$, Magdalena Raggio ${ }^{1}$, Mariana Canto ${ }^{1}$, \\ Luna Renard ${ }^{1}$, Antonello Penna ${ }^{3}$ \\ Residente, Departamento de Anestesiología y Reanimación, Hospital Clínico de la Universidad de Chile. \\ Alumno, Escuela de Medicina, Universidad de Chile. \\ Departamento de Anestesiología y Reanimación, Hospital Clínico y Facultad de Medicina de la Universidad de \\ Chile.
}

Introducción: El delirium postoperatorio (DPO) aumenta la estadía hospitalaria, la dependencia, los costos de salud y la mortalidad a largo pla$\mathrm{zo}^{1}$. La cirugía y la anestesia son eventos gatillantes para el desarrollo del DPO. Los primeros síntomas pueden ser evidenciados en la Unidad de Recuperación Postanestésica (URPA). Pese a ello, se conoce parcialmente la evolución de los pacientes con DPO que debutan en la URPA (DPO precoz $)^{2}$. Se estudió la evolución de DPO precoz en pacientes mayores de 65 años y su relación con la aparición y severidad de DPO tardío (posterior al egreso de la URPA).

Objetivo General: Determinar la incidencia de DPO precoz en pacientes mayores de 65 años sometidos a cirugía electiva con anestesia general y/o neuroaxial y describir su relación con la aparición y severidad de DPO tardío.

Material y Métodos: Se reclutaron pacientes mayores de 65 años con consentimiento firmado para realizar un estudio prospectivo, longitudinal y observacional en el HCUCH. Se excluyeron pacientes ASA III-V, con antecedentes de enfermedad neurológica, deterioro cognitivo de origen médico, enfermedad psiquiátrica mayor y pacientes con delirium previo a la cirugía. El diagnóstico de DPO se hizo con el cuestionario Confussion Assessment Method (CAM) y la severidad se determinó con CAM-S. Estos cuestionarios se aplicaron previo a la cirugía, en la URPA una vez que el paciente alcanzara un puntaje de Aldrete $\geq 9$ y luego dos veces al día hasta el $5^{\circ}$ día postoperatorio o hasta el alta. Además, se estudiaron otras variables como comorbilidades, escolaridad, tipo y duración de la cirugía, entre otras. Las variables paramétricas y no paramétricas se analizaron con la prueba $t$ de student y Mann-Withney, respectivamente. Las categóricas con el test exacto de Fisher. Un p < 0,05 fue considerado como significativo.

Resultados: Se reclutaron 91 pacientes de edad promedio de 73,0 $\pm 7,1$ años, $42,9 \%$ de sexo masculino y cuyas comorbilidades más frecuentes fueron: HTA 60,0\%, DM2 20,0\% y dislipidemia $21,1 \%$. Anestesia general fue utilizada en un $41,8 \%$, neuroaxial en un $41,8 \%$ y mixta en un $16,5 \%$. Las cirugías fueron predominantemente abdominales $(33,0 \%)$ y urológicas $(23,1 \%)$. La duración de las cirugías fue de 1,8 h (rango 0,3-7 h). Un 47\% de los pacientes tenía un nivel educacional de enseñanza media o superior. Se observó que $5(5,5 \%)$ y $7(7,7 \%)$ pacientes desarrollaron DPO precoz y tardío, respectivamente. El $60 \%$ de los que desarrollaron DPO precoz presentaron DPO tardío $(\mathrm{RR}=12,9,3,9-42,6)$. Por el contrario, sólo un $4,9 \%$ de los que no cursaron con DPO precoz evolucionaron con DPO tardío. Dos pacientes presentaron DPO severo, uno de los cuales tuvo DPO precoz. Finalmente, se determinó que una mayor edad, un menor IMC y cirugías más prolongadas se asociaron al desarrollo de DPO.

Conclusiones: La incidencia observada de DPO es inferior a lo publicado. No obstante, se pudo determinar que al presentar DPO en la URPA incrementa el riesgo de cursar con DPO tardío. Además, los pacientes añosos, más delgados y que se someten a cirugías más largas están más predispuestos a presentar DPO.

\section{Referencias}

1. Rev. Chilena de Cirugía 2012;64(3):297-305.

2. Anesth Analg 2013;117:471-8. 